\title{
Role of response availability in forgetting: A re-appraisal
}

ETHEL WEISS $\mathbf{S}^{1,2}$ BARD COLLEGE

Saltz E. Youssef's (1964) hypothesis that the loss of response differentiation is a critical factor in forgetting was confirmed, contrary to thcir own data. Response retraining of CVC's and adjectives before recall facilitated retention as compared to pairs with non-retrained items. Stimulus retraining impeded recall. The results are attributed to neither warm up nor rehearsal but rather to increased response availability. Extension of Inderwood \&, Schulz's (1960) two phase acquisition process to forgetting appears warranted.

Saltz \& Youssef (1964) hypothesized that the loss of response differentiation is a critical factor in forgetting. They a.ttempted to test this in a paired-associates situation by retraining the response term prior to a retention test. Since retention was not facilitated, they concluded that loss of response items is not an important factor in paired-associates forgetting. This conclusion is at variance with prediction from the two phase learning view (Underwood \& Schulz, 1960) and, furthermore, must be viewed cautiously because the responses retrained were words. Words, initially highly differentiated and readily available, may provide an insensitive test of a two phase forgetting theory. In this experiment an attempt was made to retrain novel response items (nonsense syllables), as a further test of the initial Saltz \& Youssef (1964) hypothesis.

\section{Method}

Design. Since the procedure used in this experiment differed from that of Saltz \& Youssef (1964), it was decided to attempt to replicate their findings. Consequently, while half the Ss were trained on adjectivenonsense syllable (A-NS) pairs, the remainder were trained on nonsense syllable-adjective (NS-A) pairs. All Ss received four training trials on the acquisition lists followed by one test trial. One hr. and $40 \mathrm{~min}$. later all Ss received a retraining treatment immediately followed by a retention test.

For half of each group (NS-A, A-NS) the retraining treatment consisted of stimulus retraining and for the other half response retraining. If retention is facilitated by rehearsal (partial reinforcement, e.g., Goss et al, 1962), then the stimulus retrained groups should do as well as, or better than, the response retrained groups, since stimulus retraining (SR) may provide a better opportunity for rehearsal than response retraining (RR).

In order to control for general warm-up effects each $S$ received retraining on half the pairs and no retraining on the remaining pairs. Thus, there were four experimental groups, Group 1 (SR, A-NS), Group 2 (SR, NS-A), Group 3 (RR, A-NS) and Group 4 (RR, NS-A).
Since retrained, non-retrained pairs were counterbalanced across lists, this led to two subgroups for each condition.

Material. Ten pairs of adjectives and nonsense syllables (Saltz and Youssef's second list) served as the materials. Since a group training and testing procedure was used, the lists were presented on index cards. For acquisition each $\mathrm{S}$ received a pack of 51 index cards, blank side up. Each S-R pair was typed on a separate card. The lists were presented in four different orders per S. Following the last pair in the list a blank card appeared. This was followed by the 10 stimulus cards on which $\mathrm{S}$ was to fill in the missing response item.

During the retraining and retention phase each $\mathrm{S}$ received a pack of 31 index cards, blank side up. Each of the five items for retraining was typed on a separate card. They appeared in four different orders. The last retraining item was followed by a blank card and then the 10 stimulus cards on which Swas to fill in the missing response item.

Procelure. Prior to acquisition Ss were instructed to read the pairs to themselves, turning to expose a pair only when E said "turn." They were told that after they had seen the pairs several times a blank card would appear. They would then be tested for learning. When E said "turn" they were to turn their card and fill in the missing item. E said turn every $5 \mathrm{sec}$. during the training and testing phase of acquisition.

One hr. and $40 \mathrm{~min}$. later, towards the end of the class session, E returned and asked the Ss to continue the experiment. They were told that they would see some of the same items they had seen before and they were just to read them to themselves, turning only when $\mathrm{E}$ said "turn." Three sec. was allowed for reading each single item. After they reached the blank card they were stopped and told they would now have the same test as they had had earlier, and that they were again to fill in the missing blanks. They were allowed $5 \mathrm{sec}$. between instructions to turn in the recall phase.

Assignment of Ss. The packs of cards were arranged in subsets of eight (four experimental conditions, counterbalanced) which were distributed to students in the order that they were seated in. Four different psychology classes, with a total enrollment of 65 were used. Since not all classes were composed of multiples of eight, the data for nine Ss was randomly dropped in order to preserve the counterbalancing. Thus a total of $14 \mathrm{Ss}$ was used in each of the four experimental conditions. Each condition was run in each class to avoid group effects. 
Table 1. Mean Number of Correct Responses for Retrained and Non-Retrained Pairs

Group 1 Group 2 Group 3 Group 4

(SR, A-NS) (SR, NS-A) (RR, A-NS) (RR,NS-A)

\begin{tabular}{lllll}
\hline Retrained Pairs & 1.93 & 1.93 & 2.71 & 2.64 \\
Non-Retrained Pairs & 2.57 & 2.36 & 2.07 & 1.57 \\
\hline
\end{tabular}

\section{Results}

The groups did not differ from each other in the number of correct responses in acquisition ( $F$ for NS-A, A-NS lists < 1, F for S, R item-to-be-retrained = $1.84, \mathrm{df}=1 / 52, p>.05)$. Contrary to expectations no more correct adjective responses were obtained than nonsense syllable responses.

Table 1 summarizes the mean number of correct responses for retrained and non-retrained pairs. An analysis of variance of this data yielded only one significant $F$. The interaction between $S$ or $R$ retraining by retrained, non-retrained pairs is significant ( $F=$ 19.00, $\mathrm{df}=1 / 52, \mathrm{p}<.01$ ). Response retrained pairs are significantly superior to $S$ retrained pairs (MannWhitney $\mathrm{U}=499.5, \mathrm{z}=1.80$, with a 1 tailed test, $\mathrm{p}<.01$ ) Response retraining facilitated recall for both the NS-A pairs and the A-NS pairs. Comparing $S$ retrained pairs to their own non-retrained pairs, it is seen that retraining of the stimulus retards correct anticipations (Wilcoxon matched pairs test, $\mathrm{T}=14, \mathrm{~N}=16, \mathrm{p}<.01$ ). Alternatively, comparing the $\mathrm{R}$ retrained pairs to their own non-retrained pairs, it is seen that response pairs yielded more correct responses (Wilcoxon matched pairs test, $\mathrm{T}=25, \mathrm{~N}=18, \mathrm{p}<.01$ ). The difference between the non-retrained pairs in the $S$ and $R$ retrained groups is not significant (Mann-Whitney $U=491.5$, $z=1.68$, with a 2 tailed test, $p>.05$ )。

\section{Discussion}

It appears that the effect of $R$ retraining is on $R$ item availability rather than being due to warm-up or rehearsal. If the effect were due to warm-up then the retrained and non-retrained pairs would not have differed from each other. If the effect were due to opportunity for rehearsal, provided by the retraining procedure, then the $S$ retrained pairs would have provided at least as much opportunity for rehearsal as the $\mathbf{R}$ retrained pairs and would not have resulted in fewer correct responses in the retention test.

It had been hypothesized that $R$ retraining would facilitate the recall of pairs with NSR's. While this was confirmed, it was also found, contrary to Saltz \& Youssef's (1964) data, that retraining adjective responses also facilitated recall. However, with the test procedure used in this study ( $5 \mathrm{sec}$. to write the $\mathbf{R}$ term and turn the card) NS-A pairs were more difficult than anticipated. Adjective responses were no more frequent in acquisition than nonsense syllable responses. Thus it appears that when $R$ items are difficult (due either to novelty or the brevity of the response interval) response retraining will facilitate recall. By increasing the availability of the response, the latency of the response should also decrease, allowing for more correct responses in a given interval.

While it is still unclear as to exactly what conditions lead to response item loss, Saltz \& Youssef's (1964) conclusion that response differentiation was not a critical factor in the forgetting of paired-associates must be questioned. Certainly, if acquisition can be described as a two phase process, it is reasonable to assume forgetting can affect these phases differentially. The data reported here are consistent with this interpretation.

\section{References}

Goss, A. E., Nodine, C. F., Gregory, B. N., Taub, H. A., \& Kennedy, K. E. Stimulus characteristics and percentage of occurrence of response members in paired-associate learning. Psychol. Monogr., 1962, 76, No. 12 (Whole No. 531 ).

Saltz, E., \& Youssef, $\boldsymbol{Z}$. I. Role of response differentiation in forgetting. J. exp. Psychol., 1964, 68, 307-311.

Underwood, B. J., \& Schulz, R. W. Meaningfulness and verbal learning. New York: J. B. Lippincott, 1960.

\section{Notes}

1. This study was conducted while the author was a recipient of an NSF Academic Year Extension Grant.

2. I wish to thank Frank Oja for many helpful comments. 